\title{
MULTI-DIMENSIONAL INDOOR LOCATION INFORMATION MODEL
}

\author{
Qing Xionga ${ }^{\text {a }}$ Qing Zhu ${ }^{\text {b,a }}$, Sisi Zlatanova ${ }^{c}$, Liang Huang ${ }^{\text {a }}$, Yan Zhou ${ }^{\text {d,c }}$, Zhiqiang Du ${ }^{\text {a }}$
}

\author{
${ }^{a}$ State Key Laboratory of Information Engineering in Surveying Mapping and Remote Sensing, Wuhan University, \\ 430072, Wuhan, P.R China, Email: whuxiong@whu.edu.cn \\ ${ }^{\mathrm{b}}$ Faculty of Geosciences and Environmental Engineering of Southwest Jiaotong University, 611756, Chengdu, Sichuan, \\ P.R.China, Email: zhuq66@263.net \\ ${ }^{\mathrm{c}}$ GISt, OTB, Delft University of Technology, Jaffalaan 9, 2628 BX Delft, The Netherlands, Email: \\ s.zlatanova@tudelft.nl \\ ${ }^{\mathrm{d}}$ School of Resources and Environment, University of Electronic Science and Technology of China, 6117569, Chengdu, \\ Sichuan, P.R.China, Email: zhouyan_gis@uestc.edu.cn
}

\section{Commission IV/WG IV/7}

KEY WORDS: Indoor, Location Model, Multi-dimensional, Indoor Navigation

\begin{abstract}
:
Aiming at the increasing requirements of seamless indoor and outdoor navigation and location service, a Chinese standard of Multidimensional Indoor Location Information Model is being developed, which defines ontology of indoor location. The model is complementary to 3D concepts like CityGML and IndoorGML. The goal of the model is to provide an exchange GML-based format for location needed for indoor routing and navigation. An elaborated user requirements analysis and investigation of state-of-the-art technology in expressing indoor location at home and abroad was completed to identify the manner humans specify location. The ultimate goal is to provide an ontology that will allow absolute and relative specification of location such as "in room 321 ", "on the second floor", as well as, "two meters from the second window", "12 steps from the door".
\end{abstract}

\section{INTRODUCTION}

Research in mobile navigation has increased in the last two decades motivated by the ever-growing use of mobile devices. Location-based services (LBSs) ${ }^{[1]}$ have attracted the interest of both academy and industry. The added value of these services is that they can provide information relevant for the location of the mobile users ${ }^{[2]}$. And human cognitive spatial representation and spatial communication are known to be hierarchic ${ }^{[3]}$. Hierarchy can covey a scene of place and of orientation, and support navigation ${ }^{[4]}$. However, location-based service on mobile devices still utilize traditional map with contextual noise. Especially for indoor environment, the hierarchic spatial information needs to be provided to users.

This paper studies the representation of indoor location to users of indoor environment. Providing such representation is challenging for absolute location and relative location have to be considered:

Absolute Location: A point in geographic space that is measured with respect to the origin of a standard coordinate system.

Relative Location: A position that is measured or described with respect to another location, not the origin of the coordinate system.

Also the indoor location should represents the functional or organizational meaning of the location. For example, the room label in reveals a location ["Room 311"], organization ["LIESMARS"] and function ["Public Computer Room"].

Combined with the hierarchical representation of indoor space that was descripted in LOD4 building model of CityGML, a Indoor Location Model that account for the Absolute Location and Relative Location is pre-requisite to generate meaningful indoor location information. The hypothesis of this paper is that nonrepresentational indoor location can be formally described by qualitative and quantitative. In the following, we first present related work and develop a model of indoor location that adheres to the demanded description of a location: Absolute Location and Relative Location. Then we design a simple indoor navigation experiment to give an example of how to use this indoor location model in indoor environment application.

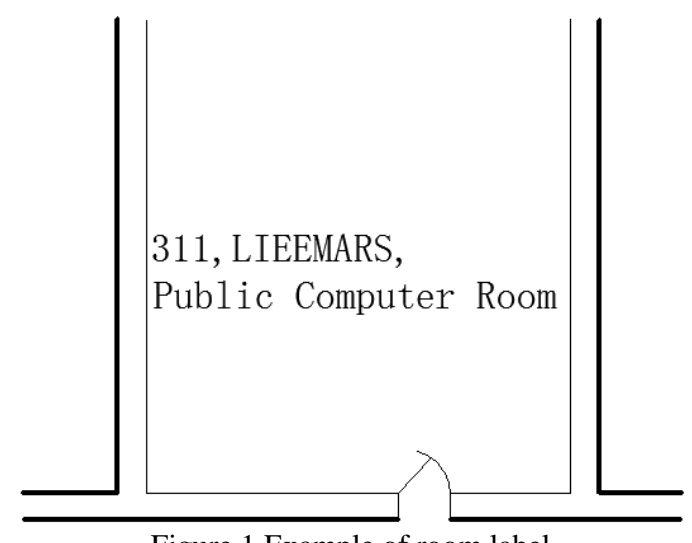

Figure 1 Example of room label 


\section{REPRESENTATION OF INDOOR LOCATION}

Today's gazetteers (place name directories) collect communally recognized place names together with their types and a georeference, typically in the form of a point ${ }^{[9]}$. However, the observation that indoor spaces differ from urban street spaces is acknowledged in other approaches as well. In particular, movement in indoor environment is richer than a linear network representation suggest ${ }^{[3]}$.

$\mathrm{Li}^{[5]}$ uses a topology-based sematic location model attached to an exit-location space model to preserve the topology and distance semantics between locations. His representation remains at one level of indoor location. UBGI and Address Standards ${ }^{[6]}$ describes a detailed location information of outdoor, but lack of indoor location defining. In the following, we will define a similar model to define indoor location.

\subsection{Indoor Location Model}

Presently, an explicit indoor location information is outside the scope of CityGML and IndoorGML.It is necessary to develop Multi-dimensional Indoor Location Model. The UML class diagram is shown in Figure 3.

Indoor Location is an abstract class, the root of indoor location. As required by real-time navigation, all the location in the indoor environment needs to be assigned a property of lifecycle. So the class of Life Circle, based on TM_Reference System ${ }^{[7]}$ introduce in ISO 19108, is associated with Indoor Location. Based on Indoor Location, there are two general ways to define indoor location in details: Indoor Absolute Location and Indoor Relative Location.

The Indoor Absolute Location allows to define a point or a cell in geographic space that is measured with respect to the origin of a standard coordinate system. There are Geometrical Absolute Location and Semantic Absolute Location aggregated by the Indoor Absolute Location. The Geometrical Absolute Location contains the description of cell location by Range of Spatial Cell Location, the Spatial reference System, a typical Cartesian Coordinate System, and the Geometrical Coordinate. The Range of Spatial Cell Location is defined by start location and end location, which were defined by Spatial Cell Location defined by the index of a octree and the Spatial Granularity ${ }^{[8]}$. The Semantic Absolute Location defines a location by the address and the attribute of location, including the information of floor, owner and description etc. For example, a description of indoor absolute location as $(100,100,10)$, containing the visible geometrical XYZ coordinates to define a location and invisible information of system parameters of the Cartesian coordinate system. Similarly a description as (A,B,C,1), containing the visible index of a octree and invisible parameters of a octress. A description as (325, 3, Lily, Office Room), containing the address and attribute information of a room's location.

The Indoor Relative Location is more complicated than the Indoor Absolute Location. It distinguishes between Geometrical Relative Location and Semantic Relative Location. The Geometrical Relative Location contains a list of Geometrical Reference Location, which defines the reference object and referenced object, which are realized by the Geometrical Reference Object with information of a Geometrical Absolute Location and description, the Value of Distance which defines the unit and value of a distance, the Value of Direction which defines the angle and reference direction of a direction. The Semantic Relative Location includes a list of Semantic Reference Location. The Location of Semantic Reference is aggregated by the Semantic Reference Object, which is realized by the Semantic Absolute Location with information of a Semantic Absolute Location and description, the Relation of Distance which includes the value of distance and distance description, the Relation of Direction which includes the value of direction and direction description, the Relation of Topology which defines adjoin, inside, intersection and link, the Relation of Indoor Location which defines down, left, right and up with a third referenced object. For example, a relative location description formalized as (Distance, Direction, Room 209, Room 210), defining the relative location from Room 209 to Room 325. And as description like "Room 210 is not far (2 feet), in the north direction, adjoining Room 209", containing the relation of distance(clear or vague expression), the relation of direction(absolute or relative expression), the relation of topology, and two Semantic Reference Object to define the relative location from Room 209 to Room 210.

\subsection{Use case of Indoor Location Model}

Knowing how to specify location (i.e. the location model presented here), the 3D building model (e.g. LOD4 building model of CityGML) and a model representing sensors, it is possible to identify the location of users in the indoor environment. In order to meet requirements of a large variety of applications, the indoor applications cloud use the 3D spaces of the building, the indoor location, and the user analysis methods provided by Indoor Service Provider as telecommunications company. The 3D spaces of the building are defined as room, corridor, window, door, doorway, staircase, elevator, furniture etc. The user analysis methods support for active pushing location service of interest to the users. For example, either indoor navigation apps on smart phone or desktop application, individual users can be navigated or they can be provided with different services in a similar manner as outdoors. In case of business-oriented applications (such as shops, hospitals), more complex spatiotemporal and location services can be provided. Yet another case can be distinguished for building managers, who need even more elaborated information about the structure of the building, furnishment, utility networks etc. The use case diagram for Indoor Location Model is shown in.

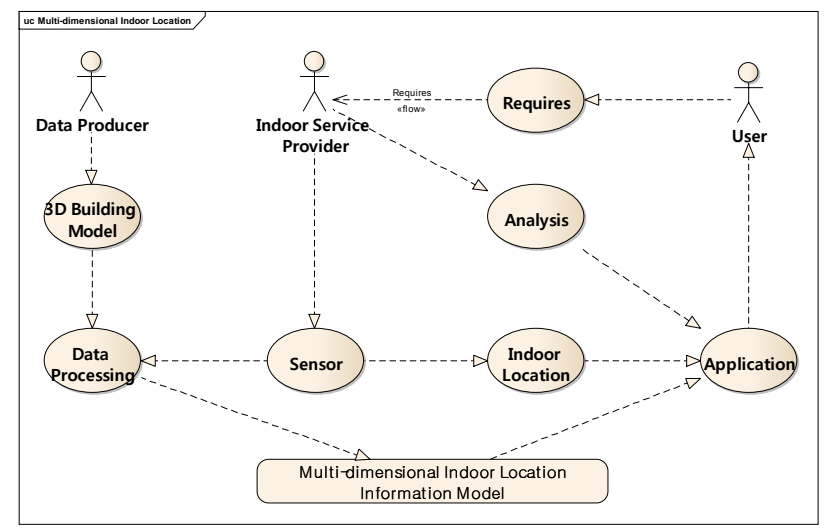

Figure 2 Use case Diagram for Indoor Location Model

\section{EXAMPLE OF USING INDOOR LOCATION}

This section illustrates a simple indoor navigation with using the indoor location model proposed above. Figure 4 shows the 
The International Archives of the Photogrammetry, Remote Sensing and Spatial Information Sciences, Volume XL-4/W4, 2013 ISPRS Acquisition and Modelling of Indoor and Enclosed Environments 2013, 11 - 13 December 2013, Cape Town, South Africa

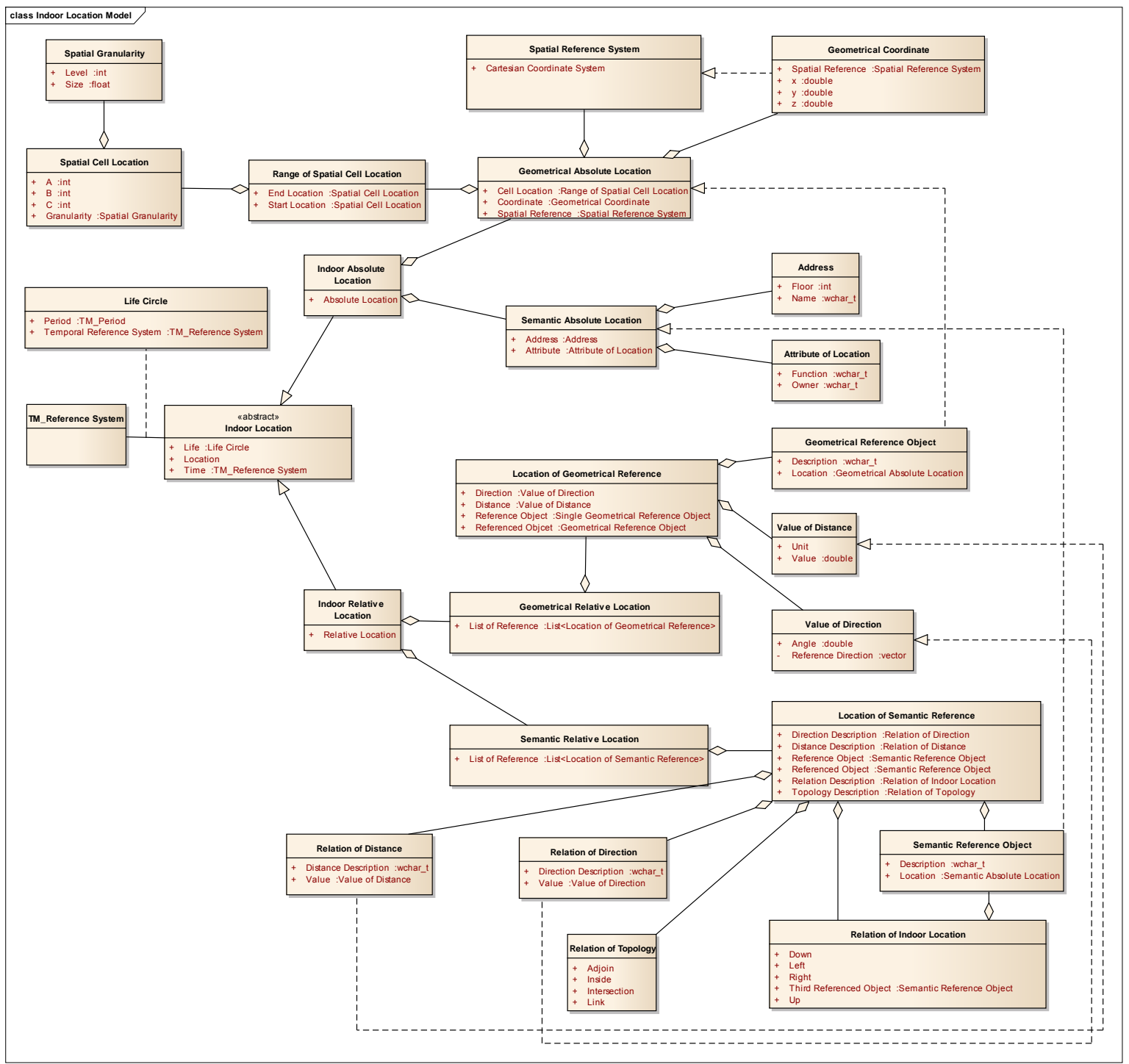

Figure 3 Class diagram of Indoor Location

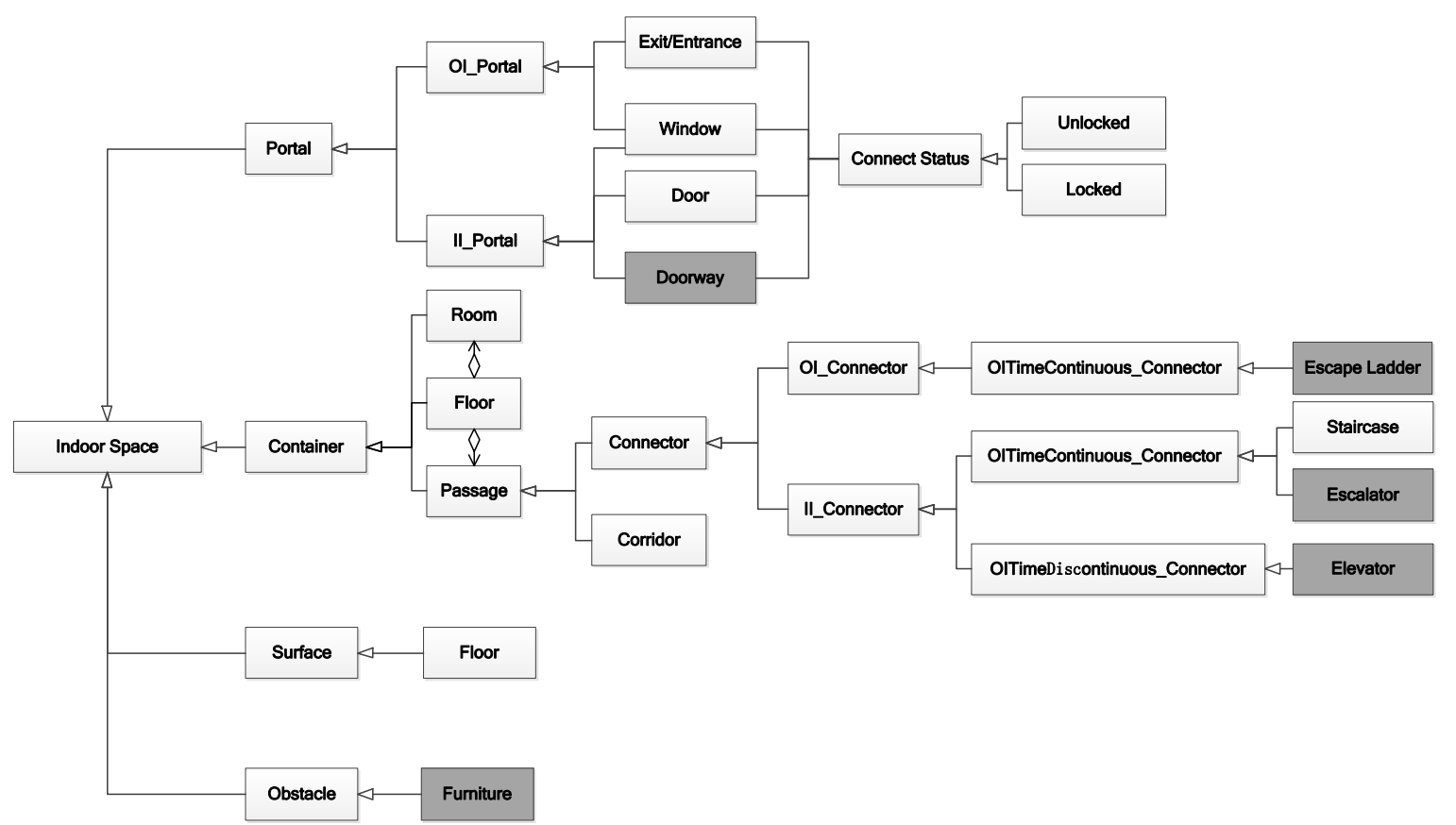

Figure 4 The structure defining of building model 
The International Archives of the Photogrammetry, Remote Sensing and Spatial Information Sciences, Volume XL-4/W4, 2013 ISPRS Acquisition and Modelling of Indoor and Enclosed Environments 2013, 11 - 13 December 2013, Cape Town, South Africa
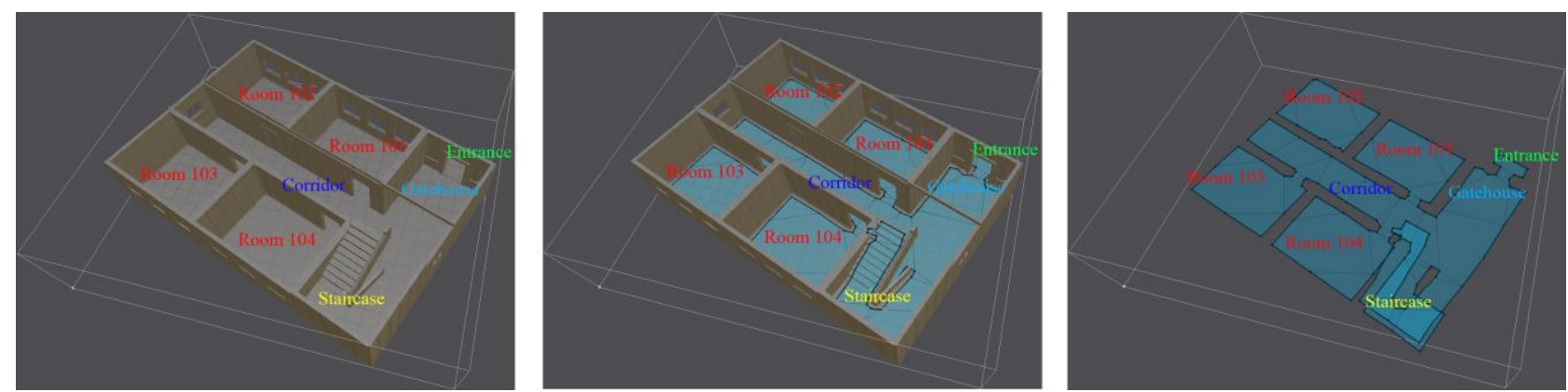

Figure 5 The experiment building model and navigation mesh
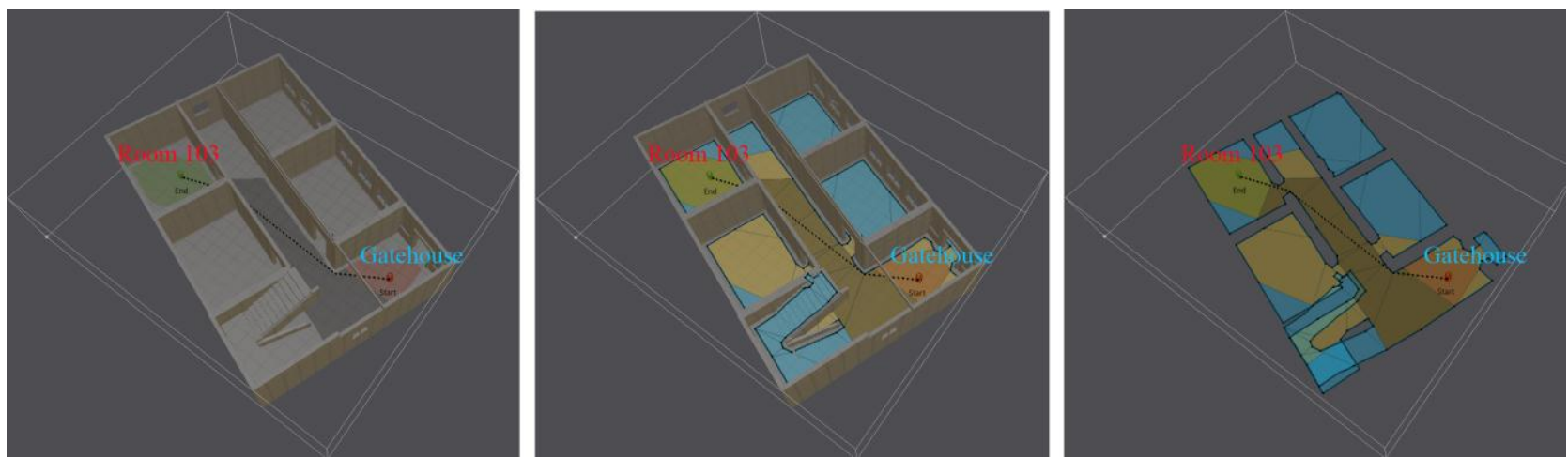

Figure 6 Navigation route from Gatehouse to Room 103
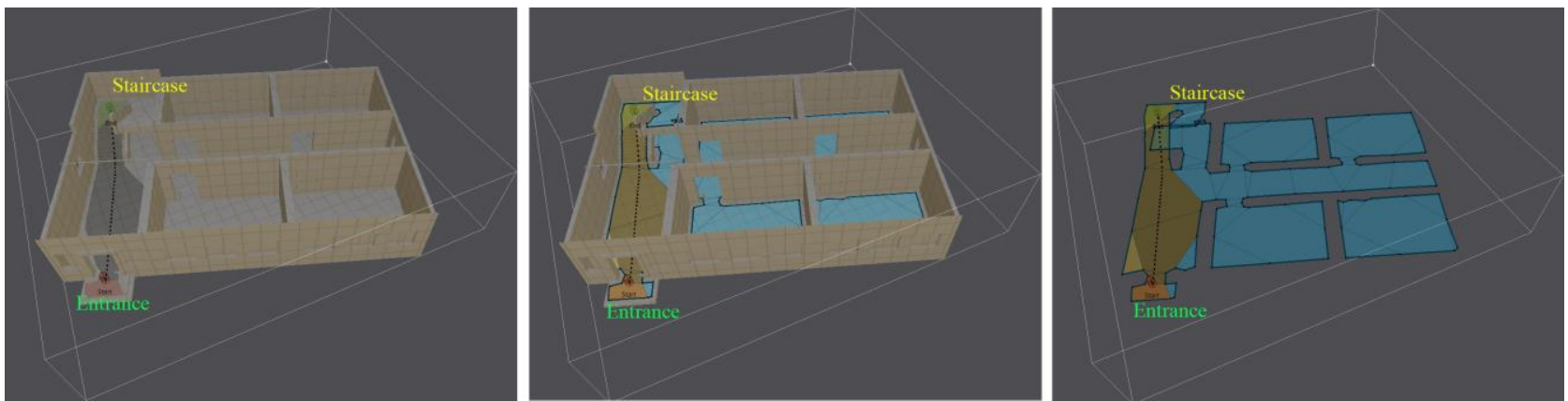

Figure 7 Navigation route from Entrance to Staircase
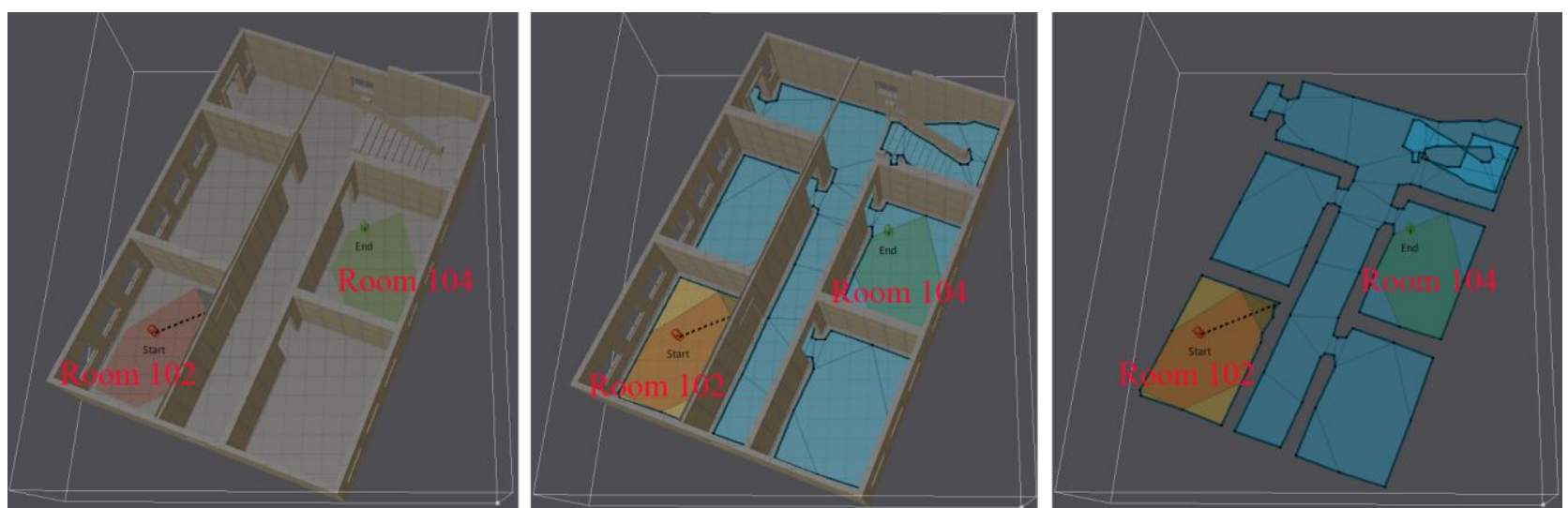

Figure 8 Influence of navigation route by a closed door 
structure defining of the example building. Indoor space is divided to four part: portal, container, surface and obstacle.

The portal could be OI_Portal (a portal that connect outdoor environment and indoor environment), such as Exit/Entrance or window, and II_Portal (a portal that connect between indoor space), for example window, door, or doorway. And all the portals have a connect status: locked means the portal could not be used or passed, and unlocked means the portal could be used or passed.

The container is the main component of a building. It contains room, passage and floor. The floor is composited by room and passage. And the passage could be a corridor or a connector. The connector could be OI_Connector (a connector that connect outdoor environment and indoor environment), such as escape ladder which is a time continuous connector, or II_Connector (a connector that connect between indoor space), such as staircase and escalator which are time continuous connectors, and elevator which is a time discontinuous connector.

The surface is just the floor, and the furniture is the obstacle.

In this experiment building, we do not create the dark parts. And the example is based on the limited parts: window, door, staircase, room, corridor and floor. Figure 5 shows the input building model, and the navigation mesh created based on the model. And during this process, we tag the room, corridor, door, staircase, floor and room. Table 1 enumerates the location information of this building.

\begin{tabular}{|c|l|}
\hline Code & Semantic Information \\
\hline 1 & Entrance \\
\hline 2 & Gatehouse \\
\hline 3 & Corridor \\
\hline 4 & Room 101 \\
\hline 5 & Room 102 \\
\hline 6 & Room 103 \\
\hline 7 & Room 104 \\
\hline 8 & Staircase \\
\hline
\end{tabular}

Table 1 Location information of experiment building

Table 2 enumerates the relation of topology of indoor location. The symbol " $\sqrt{ }$ " means the relation between the locations is adjoining. Similarly, symbol " $\times$ " means disjoining.

\begin{tabular}{|c|c|c|c|c|c|c|c|c|}
\hline & 1 & 2 & 3 & 4 & 5 & 6 & 7 & 8 \\
\hline 1 & - & $\sqrt{ }$ & $\times$ & $\times$ & $\times$ & $\times$ & $\times$ & $\times$ \\
\hline 2 & $\sqrt{ }$ & - & $\sqrt{ }$ & $\times$ & $\times$ & $\times$ & $\times$ & $\times$ \\
\hline 3 & $\times$ & $\sqrt{ }$ & - & $\sqrt{ }$ & $\times$ & $\sqrt{ }$ & $\sqrt{ }$ & $\sqrt{ }$ \\
\hline 4 & $\times$ & $\times$ & $\sqrt{ }$ & - & $\times$ & $\times$ & $\times$ & $\times$ \\
\hline 5 & $\times$ & $\times$ & $\times$ & $\times$ & - & $\times$ & $\times$ & $\times$ \\
\hline 6 & $\times$ & $\times$ & $\sqrt{ }$ & $\times$ & $\times$ & - & $\times$ & $\times$ \\
\hline 7 & $\times$ & $\times$ & $\sqrt{ }$ & $\times$ & $\times$ & $\times$ & - & $\times$ \\
\hline 8 & $\times$ & $\times$ & $\sqrt{ }$ & $\times$ & $\times$ & $\times$ & $\times$ & - \\
\hline
\end{tabular}

Table 2 Relation of topology

Based on the semantic information and navigation mesh, we set a start location, Gatehouse, with a geometrical relative location referenced to the local coordinate system of the input model. Then we set an end location, Room 103, with a geometrical relative location, too. Then we get a navigation route from start location to end location by $A^{*}$ algorithm showed in Figure 6
Similarly, Figure 7 shows a navigation route from start location, Entrance, to end location, staircase. And Figure 8 shows the influence of navigation route by a closed door.

\section{CONCLUSION AND FUTURE WORK}

This paper argues for an absolute and relative representation of indoor location. Existing indoor location service may be providing contextual noise and lack of users-concerned information about location. However the work of multidimensional indoor location information model will enrich the improvement of indoor navigation. It is able to capture geometrical location and semantic location of indoor environment of different user groups and information needed for different tasks. The proposed indoor location model is well suited for different kinds of communication of indoor location.

One task for future work is the extension and improvement of the indoor location model. This comprise the National Standard of Multi-dimensional Indoor Location Information promulgate based on this Indoor Location Model in the next year. And based on this standard, examples of multi-dimensional location and indoor navigation will be realized with indoor position technology. Along this line, the indoor location model for switching from a abstracted defining to a practical application may need to be experimented in different environment. It can be expected that defining and approaches to meet the indoor navigation of different users can be improving in an estimated future.

\section{ACKNOWLEDGMENTS}

This work is supported by National Science and Technology Support Program of China (No.2012BAH35B00), National Natural Science Foundation of China (No. 41101354), and Public Service Industry Research (No. 2014424288). The supports are gratefully acknowledged.

\section{REFERENCE}

[1]Schiller, J. and Voisard, A., 2004. Location based services. Burlington, MA: Morgan Kaufmann.

[2] Bernad J, Bobed C, Mena E, et al., 2013. A formalization for semantic location granules. International Journal of Geographical Information Science, 27(6), pp1090-1108.

[3]S. C. Hirtle and J. Jonide, 1985. Evidence of hierarchies in cognitive maps. Memory and Cognition, 13(3), pp208-217.

[4]Kai-Florian Richter, Stephan Winter and Sigit Santosa, 2011, Hierarchical Representations of Indoor Spaces. Environment and Planning B: Planning and Design, 38, pp1052-1070. [5]Li, D, Lee, D.L, 2008, A Topology-based Semantic Location Model for Indoor Applications. The Proceedings of ACM GIS. [6]Hong, S.K, 2008. Ubiquitous Geographic Information(UBGI) and Address Standards. ISO Workshop on Address Standards: Considering the issues Related to an International Address Standard, ,pp28-37.

[7] ISO T C. 211 SC, 2002: ISO 19108. Temporal schema.

[8] Richter D, Winter S, Richter K F, et al., 2013. Granularity of locations referred to by place descriptions. Computers, Environment and Urban Systems, 41, pp88-99.

[9] Hill, L. L., 2006. Georeferencing: The geographic associations of information. Cambridge, MA: Digital Libraries and Electronic Publishing, The MIT press. 